\title{
Conductas asociadas a los trastornos de pica, rumiación y evitación/restricción de alimentos en adolescentes
}

\section{Behaviors associated to the disorders of pica, rumination, and avoidant/restrictive food intake in adolescents}

\author{
Karla Areli Medina-Tepal, Rosalía Vázquez-Arévalo, \\ Xóchitl López-Aguilar y Juan Manuel Mancilla-Díaz
}

\author{
Universidad Nacional Autónoma de México ${ }^{1}$
}

Autor para correspondencia: Karla Areli Medina-Tepal,tepalkarla@gmail.com.

\begin{abstract}
RESUMEN
En la quinta versión del Manual Diagnóstico y Estadístico de los Trastornos Mentales (DSM-V) se hacen modificaciones importantes al capítulo relativo a los trastornos de la conducta alimentaria, actualmente conocidos como trastornos de la conducta alimentaria y de la ingesta de alimentos, una de las cuales es que los trastornos que se creían específicos de la infancia pueden aparecer en cualquier etapa de la vida. Por tal motivo, el objetivo del presente estudio fue identificar la ocurrencia de conductas asociadas a los trastornos de pica, rumiación y evitación/restricción de la ingesta de alimentos en adolescentes. Participaron 292 estudiantes de sexo masculino y femenino, con edad promedio de 14.58 años, que contestaron el cuestionario CuPREA. Los resultados indican que una cuarta parte de la muestra afirmó consumir sustancias no comestibles, muy pocos admitieron regurgitar, y otra cuarta parte dijo que evitaban alimentos por sus características organolépticas. En conclusión, se puede afirmar que en la adolescencia aparecen conductas relacionadas a los trastornos señalados, por lo que se abre una importante línea de investigación para caracterizar dichos trastornos en los adolescentes.
\end{abstract}

Palabras clave: Pica; Trastorno de rumiación; Trastorno de evitación/restricción de ingesta de alimentos; Adolescentes; DSM-5.

\begin{abstract}
The fifth version of the DSM, contains key modifications to the previously called "eating disorders", currently classified as feeding and eating disorders. One such modification is that disorders believed to be specific to childhood may appear at any stage of life. Thus, the objective of the present study was to identify behaviors related to the disorders of pica, rumination and avoidant/restrictive food intake disorder in adolescents. A total of of 292 male and female students with average age of 14.58 years answered the CUPREA questionnaire. Results revealed that one fourth of participants mentioned consuming inedible substances and very few admitted regurgitation. Another fourth of participants indicated that they avoided food due to its organoleptic characteristics. We therefore conclude that behaviors related to such disorders actually occur during adolescence. Thus, an important line of research opens in order to characterize these disorders in adolescents.
\end{abstract}

Keywords: Pica; Rumination disorder; Avoidant/restrictive food intake disorder; Adolescents; DSM-5.

\footnotetext{
${ }^{1}$ Grupo de Investigación en Nutrición, Facultad de Estudios Superiores Iztacala, Av. de los Barrios 1, Los Reyes Iztacala, 54090 Tlalnepantla, Edo. de México, México, tel. (55)56-23-13-33, exts.39736 y 39737, correos electrónicos: tepalkarla@gmail.com, rvamap@unam.mx, xochitll@unam.mxy jmmd@unam.mx.
} 
Recibido: 08/11/2019

Aceptado: 19/06/2020

\section{INTRODUCCIÓN}

$\mathrm{L}$ os llamados trastornos de la conducta alimentaria (TCA) fueron incluidos por primera vez en la tercera versión del Manual Diagnóstico y Estadístico de los Trastornos Men tales (DSM-III) (American Psychiatric Association [APA], 1987); sin embargo, desde ese momento hasta la fecha han experimentado diversas modificaciones de nombre y clasificación (Tabla 1).

En el DSM-V (APA, 2013) aparecen cambios relevantes respecto a la versión revisada del DSMIV (APA, 2000). Uno de ellos es que se elimina la división que había entre los trastornos de la infancia y de la adolescencia, los que se conjuntan

Tabla 1. Evolución de los trastornos de la conducta alimentaria y de la ingesta de alimentos a través de las ediciones del Manual Diagnóstico y Estadístico de los Trastornos Mentales (DSM).

\begin{tabular}{|c|c|c|c|c|c|}
\hline Publicación & DSM-III & DSM-III-TR & DSM-IV & DSM IV-TR & DSM-5 \\
\hline Año & 1980 & 1987 & 1994 & 2000 & 2013 \\
\hline \multirow[b]{2}{*}{ Capítulo } & \multirow{2}{*}{$\begin{array}{l}\text {-Trastornos } \\
\text { de inicio } \\
\text { en la infancia, } \\
\text { la niñez } \\
\text { o la adolescencia }\end{array}$} & \multirow{2}{*}{$\begin{array}{l}\text {-Trastornos } \\
\text { de inicio } \\
\text { en la infancia, } \\
\text { la niñez } \\
\text { o la adolescencia }\end{array}$} & $\begin{array}{l}\text { Trastornos } \\
\text { de la conducta } \\
\text { alimentaria }(\mathrm{TCA})^{1}\end{array}$ & $\begin{array}{l}\text { - Trastornos } \\
\text { de la conducta } \\
\text { alimentaria (TCA) }{ }^{1}\end{array}$ & \multirow{2}{*}{$\begin{array}{l}\text { - Trastornos } \\
\text { de la conducta } \\
\text { alimentaria } \\
\text { y de la ingesta de } \\
\text { alimentos (TCAIA) }\end{array}$} \\
\hline & & & $\begin{array}{l}\text {-Trastornos } \\
\text { de inicio en la } \\
\text { infancia, la niñez } \\
\text { o la adolescencia }{ }^{2}\end{array}$ & $\begin{array}{l}\text {-Trastornos } \\
\text { de inicio en la } \\
\text { infancia, la niñez } \\
\text { o la adolescencia }{ }^{2}\end{array}$ & \\
\hline \multirow[b]{2}{*}{ Nombre } & \multirow[b]{2}{*}{$\begin{array}{l}\text { - Trastornos } \\
\text { de la conducta } \\
\text { alimentaria (TCA) }\end{array}$} & \multirow[b]{2}{*}{$\begin{array}{l}\text { - Trastornos } \\
\text { de la conducta } \\
\text { alimentaria (TCA) }\end{array}$} & $\begin{array}{l}\text { Trastornos } \\
\text { de la conducta } \\
\text { alimentaria }(\mathrm{TCA})^{1} \\
\end{array}$ & $\begin{array}{l}\text { - Trastornos } \\
\text { de la conducta } \\
\text { alimentaria }(\mathrm{TCA})^{1}\end{array}$ & \multirow[b]{2}{*}{$\begin{array}{l}\text { - Trastornos } \\
\text { de la conducta } \\
\text { alimentaria } \\
\text { y de la ingesta de } \\
\text { alimentos (TCAIA) }\end{array}$} \\
\hline & & & $\begin{array}{l}\text {-Trastornos } \\
\text { de la ingestión } \\
\text { y de la conducta } \\
\text { alimentaria } \\
\text { de la infancia } \\
\text { o la niñez } \\
\end{array}$ & $\begin{array}{l}\text {-Trastornos } \\
\text { de la ingestión } \\
\text { y de la conducta } \\
\text { alimentaria } \\
\text { de la infancia } \\
\text { o la niñez }\end{array}$ & \\
\hline \multirow[b]{2}{*}{$\begin{array}{l}\text { Trastornos } \\
\text { que incluía }\end{array}$} & \multirow[b]{2}{*}{$\begin{array}{l}\text {-Anorexia nerviosa } \\
\text {-Bulimia } \\
\text {-Trastorno } \\
\text { alimenticio atípico } \\
\text {-Pica } \\
\text {-Trastorno } \\
\text { por rumiación } \\
\text { en la infancia }\end{array}$} & \multirow[b]{2}{*}{$\begin{array}{l}\text {-Trastorno } \\
\text { por atracón }{ }^{1} \\
\text {-Anorexia nerviosa } \\
\text {-Bulimia nerviosa } \\
\text {-TCANE } \\
\text {-Pica } \\
\text {-Trastorno } \\
\text { por rumiación }\end{array}$} & $\begin{array}{l}\text {-TCA específicos }{ }^{1} \\
\text {-Anorexia nerviosa }{ }^{1} \\
\text { - Bulimia nerviosa }{ }^{1} \\
\text {-TCANE }{ }^{1} \\
\text {-Trastorno } \\
\text { por atracón }{ }^{1}\end{array}$ & $\begin{array}{l}\text {-TCA específicos }{ }^{1} \\
\text {-Anorexia nerviosa }{ }^{1} \\
\text {-Bulimia nerviosa }{ }^{1} \\
\text {-TCANE }{ }^{1} \\
\text {-Trastorno } \\
\text { por atracón }^{1}\end{array}$ & \multirow{2}{*}{$\begin{array}{l}\text {-Anorexia nerviosa } \\
\text {-Bulimia nerviosa } \\
\text {-Trastorno } \\
\text { por atracón } \\
\text {-Pica } \\
\text { - Trastorno } \\
\text { de rumiación } \\
\text { - Trastorno por } \\
\text { evitación/restricción } \\
\text { de alimentos } \\
\text {-Otros trastornos } \\
\text { alimentarios } \\
\text { o de la ingestión } \\
\text { de alimentos } \\
\text { - Trastorno } \\
\text { alimentario } \\
\text { o de la ingestión } \\
\text { de alimentos } \\
\text { no específico }\end{array}$} \\
\hline & & & $\begin{array}{l}\text {-Pica }{ }^{2} \\
\text {-Trastorno } \\
\text { por rumiación }{ }^{2} \\
\text {-Trastorno } \\
\text { de la ingestión } \\
\text { alimentaria de la } \\
\text { infancia o la niñez }\end{array}$ & $\begin{array}{l}\cdot \text { Pica }^{2} \\
\text {-Trastorno } \\
\text { por rumiación }{ }^{2} \\
\text {-Trastorno } \\
\text { de la ingestión } \\
\text { alimentaria } \\
\text { de la infancia } \\
\text { o la niñez }\end{array}$ & \\
\hline
\end{tabular}

${ }^{1}$ Trastornos de la conducta alimentaria

${ }^{2}$ Trastornos de inicio en la infancia, la niñez o la adolescencia 
en una sola clasificación denominada "trastornos de la conducta alimentaria y de la ingesta de alimentos", o TCAIA, la cual incluye los trastornos de anorexia nerviosa (AN), bulimia nerviosa (BN), trastorno por atracón (TPA), pica y trastorno de rumiación y trastorno de evitación/restricción de la ingesta de alimentos (TERIA) (Vázquez, López, Tellez-Girón y Mancilla, 2015).

Según Uher y Rutter (2012), a partir de dicha modificación se podrá detectar y conocer la evolución de cualesquiera de estos trastornos en las diferentes etapas del desarrollo, y además el porcentaje de los TCANE disminuirá.

A diferencia de los trastornos de $\mathrm{AN}$ y BN, el estudio y conocimiento de los trastornos de pica, rumiación y TERIA son aún limitados, lo que tiene importantes implicaciones, una de las cuales es que se desconoce lo que ocurre con estas perturbaciones en la adolescencia debido a que su investigación se ha centrado en niños, personas con discapacidad y mujeres embarazadas (Marcus y Wildes, 2016). Además, tales padecimientos provocan diversas enfermedades (Chandran, Anderson, Kennedy, Kohn y Clarke, 2015; Chiarello, Marini, Ballerini y Ricca, 2017), e incluso la muerte (Bogdanovic, Alempijevic, Curcic y Durmic, 2018), por lo que parece indispensable profundizar más en ellos.

El pica se caracteriza por la ingesta de sustancias crudas no comestibles. Su nombre proviene de la palabra latina pica, que significa "urraca" (Pica pica) debido a que el comportamiento alimentario de esta ave se asemeja al de las personas que padecen dicha psicopatología (Byard, 2014).

El consumo de sustancias no comestibles se remonta a los antiguos griegos y romanos, quienes consumían tierra o barro para tratar enfermedades, envenenamientos e intoxicaciones (Danford, 1982). Parry-Jones y Parry-Jones (1992) afirman que en los siglos XVI y XVII este trastorno estaba relacionado con la clorosis, caracterizada por la deficiencia de hierro, la cual afectaba principalmente a mujeres púberes.

Más tarde, en los siglos XVIII y XIX, Imray (1843) reportó que esclavos sometidos a trabajos excesivos de Estados Unidos y del Caribe consumían tierra o arcilla porque la alimentación que recibían era deficiente. A este fenómeno lo denominó caquexia africana o mal d'estomac, entre cuyas complicaciones señala el cambio de temperamento, afecciones digestivas, debilidad general, dolor en la región cardiaca, dificultad para respirar, palidez y muerte. En el siglo XX la investigación de este trastorno se centró en lactantes $\mathrm{y}$ niños pequeños debido a que morían envenenados por la ingesta de objetos no comestibles que contenían grandes concentraciones de plomo, y en algunos casos se identificó que dicha conducta continuaba hasta edades avanzadas (Parry-Jones y Parry-Jones, 1992).

A su vez, el trastorno de rumiación se caracteriza por la regurgitación de alimentos parcialmente digeridos que son vueltos a masticar, para después tragarlos nuevamente o escupirlos (Birmingham y Firoz, 2006); también a este trastorno se le conoce con el nombre de mericismo. La diferencia entre ambos conceptos es que rumiación se utiliza indistintamente en humanos y animales, mientras que mericismo se emplea únicamente en humanos (Parry-Jones, 1994). Según el citado autor, los primeros casos de este trastorno se reportaron en el siglo XVII, cuando se suponía que las personas que rumiaban tenían herencia bovina (padre con cuernos), y asimismo que la madre había convivido con ganado durante el embarazo, lo cual había afectado al feto provocándole el desarrollo de ciertos atributos bovinos. En el siglo siguiente se reportaron casos de rumiación en niños y adultos, en su mayoría hombres, siendo las edades de inicio entre los cinco y quince años. A diferencia del siglo anterior, además de la herencia, atribuían dicho trastorno a la imitación, la cual favorecía su ocurrencia.

En los siglos XIX y XX se llevaron a cabo algunos estudios con infantes porque la regurgitación del alimento traía consecuencias graves para su salud, como desnutrición, disminución de la ta1la, desequilibrio electrolítico e incluso la muerte en algunos casos (Malcolm, Thumshirn, Camilleri y Williams, 1997). En 1917, Grulee propuso que este trastorno se originaba como consecuencia de la "hiperexcitabilidad de los músculos involuntarios responsables de la digestión", señalando 
además que era mucho más frecuente de lo que se suponía en aquella época, y criticando el hecho de que no fuese reconocido por los pediatras a pesar de que cobraba la vida de muchos infantes. De igual forma, se hicieron diversos estudios en personas con anorexia nerviosa (AN) y bulimia nerviosa $(\mathrm{BN})$, toda vez que estos pacientes reportaban regurgitación involuntaria y recurrían frecuentemente a esta conducta como una forma de controlar su peso (Eckern, Stevens y Mitchell, 1998).

El trastorno de evitación/restricción de la ingesta de alimentos (TERIA) se caracteriza por evitar consumir alimentos a causa de la falta de interés en alimentarse, por las características organolépticas de la comida (olor, color, sabor o textura de la comida) y por las consecuencias repulsivas de la acción de comer, como atragantarse, asfixiarse o vomitar (APA, 2013).

Existen pocos antecedentes del TERIA, probablemente porque formaba parte de los TCANE. Al respecto, Nicely, Lane-Loney, Masciulli, Hollenbeak y Ornstein (2014) señalan que el TERIA procede de las descripciones clínicas de otros trastornos, de los cuales se han extraído algunas de las características que actualmente lo conforman. Uno de estos es el trastorno emocional de evitación de alimentos, que implica que la restricción alimentaria no se debe a la preocupación por la figura o el peso corporal; otro es la alimentación selectiva, de la que emana el rasgo de la evitación de alimentos a causa de sus características organolépticas, y por último la disfagia funcional, en la cual aparece la característica del miedo a comer por las consecuencias repulsivas. Call, Walsh y Attia (2013) afirman que el TERIA ha reemplazado al trastorno de la ingestión alimentaria de la infancia o la niñez. Si bien sus criterios diagnósticos son similares, existen diferencias que complementan al TERIA, como las razones por las cuales aparece la restricción alimentaria, sus complicaciones (pérdida de peso significativa, deficiencia nutriticional, dependencia de la alimentación enteral e interferencia en el funcionamiento psicosocial) y la eliminación del criterio de edad de inicio del trastorno antes de los seis años.

\section{Prevalencia de pica, trastorno de rumiación y TERIA}

Varios estudios hechos en la población general reportan que la prevalencia de pica va de 0.02 a $76.5 \%$. Tan amplio rango puede deberse a la metodología empleada, ya que estudios con porcentajes elevados de prevalencia suelen disponer de muestras pequeñas y utilizar entrevistas personales como método de recolección de datos, mientras que estudios con baja prevalencia se efectúan en muestras grandes y empleando frecuentemente métodos pasivos, como la revisión de notas clínicas (Sturmey y Williams, 2016).

La prevalencia del trastorno de rumiación es desconocida. Según Hartman, Poulain, Vogel, Hiemisch, Kiess y Hilbert (2018), ello puede deberse a los diversos nombres con que se le conoce: "trastorno de regurgitación", "trastorno de rumiación", "síndrome de rumiación" (Mousa, Montgomery y Alioto 2014) y, según Parry-Jones (1994), "mericismo".

El TERIA, a su vez, tiene una prevalencia de 5 a $22.5 \%$ en niños y adolescentes (Fisher et al., 2014; Nicely et al., 2001; Norris et al., 2014). Se reporta una mayor prevalencia de casos de TERIA en hombres que en mujeres, y también que los pacientes suelen ser más jóvenes que los que sufren AN, quienes presentan una duración de la enfermedad más prolongada y con comorbilidades médicas o psiquiátricas (Nakai, Nin, Noma, Teramukai y Wonderlich, 2016; Nakai, Noma, Nin, Teramukai y Wonderlinch, 2015: Norris y Katzman, 2015).

\section{Conductas de pica, trastorno de rumiación y TERIA}

Si bien el estudio de estos trastornos desde la publicación del DSM-V (APA, 2013) ha aumentado, aún es muy pobre. En la actualidad se cuenta con estudios hechos en niños y adolescentes que evidencian la presencia de conductas asociadas a dichos trastornos. Uno de ellos fue el realizado por Hartmann et al. (2018), en el que $12.31 \%$ mostraba conductas de pica, más frecuentes en los varo- 
nes de menos de 10 años, mientras que en 11.94\% de los casos se confirmó la práctica de conductas de rumiación en las que no se hallaron diferencias por sexo ni por edad.

Otro estudio similar fue el elaborado por Murray, Thomas, Hinz, Munsch y Hilbert (2018), en el que 10.0 y $9.7 \%$ de los participantes manifestaron conductas asociadas a pica y rumiación, respectivamente; además, los autores afirman no haber encontrado diferencias por sexo, edad o índice de masa corporal.

Hartman (2019) evaluó el consumo de sustancias no comestibles en adolescentes y adultos, encontrando que sus 78 participantes dijeron ingerir sustancias no comestibles, aunque $47.44 \%$ señaló hacerlo con mayor frecuencia; entre lo más consumido se encuentran la comida sin cocinar o cruda, partes o fluidos corporales, pasto, hojas y papel y sustancias semejantes a la tierra. No obstante, tal práctica no es exclusiva de muestras comunitarias. Delaney et al. (2015), por ejemplo, reportaron que $7.4 \%$ de individuos con algún trastorno alimentario (AN, BN o TPA) y $4.0 \%$ de las personas obesas consumían sustancias no comestibles. En cuanto al trastorno de rumiación, los mencionados autores hallaron que cuatro pacientes de su estudio cumplieron con los criterios para su diagnóstico, aunque ninguno fue elegido para un diagnóstico formal debido a que describían su conducta como voluntaria, pues regurgitaban la comida dependiendo de su sabor, y otros no pudieron ser diagnosticados porque no podían distinguir si la regurgitación era voluntaria o se debía a algún proceso fisiológico, como por ejemplo el reflujo.

En cuanto al TERIA, Fisher et al. (2014) encontraron que niños y adolescentes con este trastorno eran clínicamente diferentes de personas con $\mathrm{AN}$ o BN en virtud de que manifestaban más ansiedad y más síntomas gastrointestinales, y además que eran más susceptibles a desarrollar otros trastornos de la alimentación en el futuro. Nicely et al. (2014) añaden que dentro de las causas de la evitación de alimentos están el miedo a padecer enfermedades como hipercolesterolemia, obesidad o diabetes, y asimismo que personas con TERIA muestran un mayor deterioro cognitivo y más problemas de carácter social.
Si bien la eliminación en la clasificación de los trastornos de la infancia y la adolescencia amplía el espectro respecto a la ocurrencia de estos trastornos en cualquier etapa del desarrollo, ello exige una investigación más profunda, en particular de las conductas y síntomas asociados a dichos trastornos. Por tanto, el objetivo del presente estudio fue identificar la presencia de conductas asociadas al pica, la rumiación y el TERIA en adolescentes mexicanos.

\section{MÉTODO}

\section{Muestra}

La muestra, no probabilística, se conformó por 292 participantes (135 de sexo masculino y 157 de sexo femenino), con edades de 12 a 19 años $(\mathrm{M}=4.58$, D.E. $=2.01$ ), de escolaridad secundaria y preparatoria, procedentes de instituciones educativas públicas de la ciudad de México.

\section{Instrumentos}

\section{Cuestionario de Pica, Rumiación}

y Evitación/restricción de Alimentos (CuPREA)

(Medina,Vázquez, López y Mancilla, 2017).

Este cuestionario tiene como propósito evaluar conductas alimentarias asociadas al pica, la rumiación y el trastorno por evitación/restricción de alimentos. Para evaluar las conductas asociadas al trastorno de rumiación, se formulan cuatro preguntas que indagan sobre la conducta de regurgitar y su frecuencia. El instrumento muestra una consistencia interna, medida con el coeficiente alfa de Cronbach, de 0.85. En cuanto al pica, se emplean quince preguntas sobre el consumo de sustancias no comestibles, crudas o no procesadas, así como su frecuencia, con un coeficiente alfa de Cronbach de 0.73 . Por último, para el TERIA se utilizan cuatro ítems cuyo coeficiente es de 0.61 , que abordan la evitación de alimentos por sus características organolépticas, las implicaciones sobre la salud que tiene esa evitación o restricción, y las causas por las cuales se produce el trastorno. 


\section{Procedimiento}

Se llevó a cabo una entrevista con los directivos de dos instituciones educativas públicas, a quienes se solicitó su autorización para llevar a cabo el estudio dentro de las instalaciones, para lo cual se les presentó el protocolo de la investigación y el instrumento que se aplicaría a los alumnos.

Una vez concedido el acceso, se realizó una junta con los padres de familia, a quienes se proporcionó la información sobre el estudio, solicitándoles su aprobación para que sus hijos participaran en ella; asimismo, se subrayó la confidencialidad de los datos proporcionados y se les presentó una carta de consentimiento informado para su firma. Finalmente, se administró el CuPREA solamente a los alumnos autorizados por sus padres.

\section{Análisis de resultados}

Los resultados se analizaron según el sexo y fase de la adolescencia (temprana, de 12 a los 14 años, y tardía, de 15 a 19 años). Además, se evaluó la ocurrencia de cada trastorno mediante una respuesta dicotómica de "sí o no" y la frecuencia de las conductas asociadas a los mismos (de un día a más de cinco veces a la semana) expresada en porcentajes.

\section{RESULTADOS}

\section{Pica}

En total, $25.68 \%$ de la muestra confirmó consumir sustancias no comestibles, de los cuales $15.41 \%$ indicó una sustancia, siendo más habitual dicho consumo en mujeres en la adolescencia temprana. Las sustancias más ingeridas fueron pasta de dientes $(7.19 \%)$, plásticos $(2.05 \%)$, papel (1.34\%) y goma de borrar $(0.68 \%)$ (Tabla 2$)$.

Tabla 2. Porcentaje de consumo de sustancias no comestibles en adolescentes mexicanos.

\begin{tabular}{|c|c|c|c|c|c|}
\hline \multirow{4}{*}{$\begin{array}{c}\text { Sustancia } \\
\text { no comestible }\end{array}$} & \multicolumn{2}{|c|}{$\begin{array}{l}\text { Mujeres } \\
(\mathrm{n}=157)\end{array}$} & \multirow{2}{*}{\multicolumn{2}{|c|}{$\begin{array}{l}\text { Hombres } \\
(\mathrm{n}=135)\end{array}$}} & \multirow{4}{*}{$\begin{array}{c}\text { Muestra } \\
\text { total } \\
(\mathrm{N}=\mathbf{2 9 2})\end{array}$} \\
\hline & \multicolumn{2}{|c|}{ Adolescencia } & & & \\
\hline & Temprana & Tardía & Temprana & Tardía & \\
\hline & $(\mathrm{n}=85)$ & $(\mathrm{n}=72)$ & $(n=66)$ & $(n=69)$ & \\
\hline Pasta de dientes & $\begin{array}{l}15.29 \% \\
(\mathrm{n}=13)\end{array}$ & $\begin{array}{l}9.72 \% \\
(\mathrm{n}=7)\end{array}$ & $\begin{array}{l}4.55 \% \\
(\mathrm{n}=3)\end{array}$ & $\begin{array}{l}7.25 \% \\
(\mathrm{n}=5)\end{array}$ & $\begin{array}{c}9.59 \% \\
(\mathrm{n}=28)\end{array}$ \\
\hline Plástico & $\begin{array}{l}2.35 \% \\
(\mathrm{n}=2)\end{array}$ & $\begin{array}{l}1.39 \% \\
(\mathrm{n}=1)\end{array}$ & $\begin{array}{c}0 \% \\
(\mathrm{n}=0)\end{array}$ & $\begin{array}{l}4.35 \% \\
(\mathrm{n}=3)\end{array}$ & $\begin{array}{l}2.05 \% \\
(\mathrm{n}=6)\end{array}$ \\
\hline Papel & $\begin{array}{l}2.35 \% \\
(\mathrm{n}=2)\end{array}$ & $\begin{array}{l}1.39 \% \\
(\mathrm{n}=1)\end{array}$ & $\begin{array}{l}1.52 \% \\
(\mathrm{n}=1)\end{array}$ & $\begin{array}{c}0 \% \\
(\mathrm{n}=0)\end{array}$ & $\begin{array}{l}1.37 \% \\
(\mathrm{n}=4)\end{array}$ \\
\hline Goma de borrar & $\begin{array}{c}0 \% \\
(\mathrm{n}=0)\end{array}$ & $\begin{array}{c}0 \% \\
(\mathrm{n}=0)\end{array}$ & $\begin{array}{l}1.52 \% \\
(\mathrm{n}=1)\end{array}$ & $\begin{array}{l}1.45 \% \\
(\mathrm{n}=1)\end{array}$ & $\begin{array}{l}0.68 \% \\
(\mathrm{n}=2)\end{array}$ \\
\hline Pintura & $\begin{array}{l}1.18 \% \\
(\mathrm{n}=1)\end{array}$ & $\begin{array}{c}0 \% \\
(\mathrm{n}=0)\end{array}$ & $\begin{array}{c}0 \% \\
(\mathrm{n}=0)\end{array}$ & $\begin{array}{c}0 \% \\
(\mathrm{n}=0)\end{array}$ & $\begin{array}{l}0.34 \% \\
(\mathrm{n}=1)\end{array}$ \\
\hline Pegamento & $\begin{array}{c}0 \% \\
(\mathrm{n}=0)\end{array}$ & $\begin{array}{c}0 \% \\
(\mathrm{n}=0)\end{array}$ & $\begin{array}{c}0 \% \\
(\mathrm{n}=0)\end{array}$ & $\begin{array}{l}1.45 \% \\
(\mathrm{n}=1)\end{array}$ & $\begin{array}{l}0.34 \% \\
(\mathrm{n}=1)\end{array}$ \\
\hline Cabello & $\begin{array}{c}0 \% \\
(\mathrm{n}=0)\end{array}$ & $\begin{array}{l}1.39 \% \\
(\mathrm{n}=1)\end{array}$ & $\begin{array}{c}0 \% \\
(\mathrm{n}=0)\end{array}$ & $\begin{array}{c}0 \% \\
(\mathrm{n}=0)\end{array}$ & $\begin{array}{l}0.34 \% \\
(\mathrm{n}=1)\end{array}$ \\
\hline Gis & $\begin{array}{l}1.18 \% \\
(\mathrm{n}=1)\end{array}$ & $\begin{array}{c}0 \% \\
(\mathrm{n}=0)\end{array}$ & $\begin{array}{c}0 \% \\
(\mathrm{n}=0)\end{array}$ & $\begin{array}{c}0 \% \\
(\mathrm{n}=0)\end{array}$ & $\begin{array}{l}0.34 \% \\
(\mathrm{n}=1)\end{array}$ \\
\hline Algodón & $\begin{array}{c}0 \% \\
(\mathrm{n}=0)\end{array}$ & $\begin{array}{c}0 \% \\
(\mathrm{n}=0)\end{array}$ & $\begin{array}{l}1.52 \% \\
(\mathrm{n}=1) \\
\end{array}$ & $\begin{array}{c}0 \% \\
(\mathrm{n}=0)\end{array}$ & $\begin{array}{l}0.34 \% \\
(\mathrm{n}=1) \\
\end{array}$ \\
\hline
\end{tabular}


Además, $6.85 \%$ de los participantes señaló consumir dos o tres sustancias no comestibles, siendo las más ingeridas el papel y pasta de dientes, así como el plástico y pasta de dientes, con $1.03 \%$, respectivamente (Tabla 3). Cabe mencionar que $3.42 \%$ de la muestra informó consumir más de cuatro sustancias no comestibles.

Tabla 3. Porcentaje de consumo de dos o tres sustancias no comestibles en adolescentes mexicanos.

\begin{tabular}{|c|c|c|c|c|c|}
\hline \multirow{4}{*}{$\begin{array}{l}\text { Consumo de dos o tres sustancias } \\
\text { no comestibles }\end{array}$} & \multicolumn{2}{|c|}{$\begin{array}{l}\text { Mujeres } \\
(\mathrm{n}=157)\end{array}$} & \multirow{2}{*}{\multicolumn{2}{|c|}{$\begin{array}{l}\text { Hombres } \\
(\mathrm{n}=\mathbf{1 3 5})\end{array}$}} & \multirow{4}{*}{$\begin{array}{c}\text { Muestra } \\
\text { total } \\
(\mathbf{N}=\mathbf{2 9 2})\end{array}$} \\
\hline & \multicolumn{2}{|c|}{ Adolescencia } & & & \\
\hline & Temprana & Tardía & Temprana & Tardía & \\
\hline & $(\mathrm{n}=85)$ & $(\mathrm{n}=72)$ & $(n=66)$ & $(n=69)$ & \\
\hline Papel y pasta de dientes & $\begin{array}{l}1.18 \% \\
(\mathrm{n}=1)\end{array}$ & $\begin{array}{c}0 \% \\
(\mathrm{n}=0)\end{array}$ & $\begin{array}{l}1.52 \% \\
(\mathrm{n}=1)\end{array}$ & $\begin{array}{l}1.45 \% \\
(\mathrm{n}=1)\end{array}$ & $\begin{array}{l}1.03 \% \\
(\mathrm{n}=3)\end{array}$ \\
\hline Plástico y pasta de dientes & $\begin{array}{c}0 \% \\
(\mathrm{n}=0)\end{array}$ & $\begin{array}{l}1.39 \% \\
(\mathrm{n}=1)\end{array}$ & $\begin{array}{c}0 \% \\
(\mathrm{n}=0)\end{array}$ & $\begin{array}{l}2.90 \% \\
(\mathrm{n}=2)\end{array}$ & $\begin{array}{l}1.03 \% \\
(\mathrm{n}=3)\end{array}$ \\
\hline Objetos de metal y papel & $\begin{array}{c}0 \% \\
(\mathrm{n}=0)\end{array}$ & $\begin{array}{c}0 \% \\
(\mathrm{n}=0)\end{array}$ & $\begin{array}{l}3.03 \% \\
(\mathrm{n}=2)\end{array}$ & $\begin{array}{c}0 \% \\
(\mathrm{n}=0)\end{array}$ & $\begin{array}{l}0.68 \% \\
(\mathrm{n}=2)\end{array}$ \\
\hline Goma, papel y pasta & $\begin{array}{c}0 \% \\
(\mathrm{n}=0)\end{array}$ & $\begin{array}{c}0 \% \\
(\mathrm{n}=0)\end{array}$ & $\begin{array}{l}3.03 \% \\
(\mathrm{n}=2)\end{array}$ & $\begin{array}{c}0 \% \\
(\mathrm{n}=0)\end{array}$ & $\begin{array}{l}0.68 \% \\
(\mathrm{n}=2)\end{array}$ \\
\hline Cabello y papel & $\begin{array}{c}0 \% \\
(\mathrm{n}=0)\end{array}$ & $\begin{array}{l}1.39 \% \\
(\mathrm{n}=1)\end{array}$ & $\begin{array}{c}0 \% \\
(\mathrm{n}=0)\end{array}$ & $\begin{array}{c}0 \% \\
(\mathrm{n}=0)\end{array}$ & $\begin{array}{l}0.34 \% \\
(\mathrm{n}=1)\end{array}$ \\
\hline Objetos de metal y pasta de dientes & $\begin{array}{c}0 \% \\
(\mathrm{n}=0)\end{array}$ & $\begin{array}{c}0 \% \\
(\mathrm{n}=0)\end{array}$ & $\begin{array}{c}0 \% \\
(\mathrm{n}=0)\end{array}$ & $\begin{array}{l}1.45 \% \\
(\mathrm{n}=1)\end{array}$ & $\begin{array}{l}0.34 \% \\
(\mathrm{n}=1)\end{array}$ \\
\hline Papel y jabón & $\begin{array}{c}0 \% \\
(\mathrm{n}=0)\end{array}$ & $\begin{array}{l}1.39 \% \\
(\mathrm{n}=1)\end{array}$ & $\begin{array}{c}0 \% \\
(\mathrm{n}=0)\end{array}$ & $\begin{array}{c}0 \% \\
(\mathrm{n}=0)\end{array}$ & $\begin{array}{l}0.34 \% \\
(\mathrm{n}=1)\end{array}$ \\
\hline Plástico y papel & $\begin{array}{c}0 \% \\
(\mathrm{n}=0)\end{array}$ & $\begin{array}{c}0 \% \\
(\mathrm{n}=0)\end{array}$ & $\begin{array}{c}0 \% \\
(\mathrm{n}=0)\end{array}$ & $\begin{array}{l}1.45 \% \\
(\mathrm{n}=1)\end{array}$ & $\begin{array}{l}0.34 \% \\
(\mathrm{n}=1)\end{array}$ \\
\hline Plástico, pegamento y fósforos & $\begin{array}{l}1.18 \% \\
(\mathrm{n}=1)\end{array}$ & $\begin{array}{c}0 \% \\
(\mathrm{n}=0)\end{array}$ & $\begin{array}{c}0 \% \\
(\mathrm{n}=0)\end{array}$ & $\begin{array}{c}0 \% \\
(\mathrm{n}=0)\end{array}$ & $\begin{array}{l}0.34 \% \\
(\mathrm{n}=1)\end{array}$ \\
\hline Cabello, papel y pasta dental & $\begin{array}{c}0 \% \\
(\mathrm{n}=0)\end{array}$ & $\begin{array}{l}1.39 \% \\
(\mathrm{n}=1)\end{array}$ & $\begin{array}{c}0 \% \\
(\mathrm{n}=0)\end{array}$ & $\begin{array}{c}0 \% \\
(\mathrm{n}=0)\end{array}$ & $\begin{array}{l}0.34 \% \\
(\mathrm{n}=1)\end{array}$ \\
\hline Plástico, papel y pasta & $\begin{array}{c}0 \% \\
(\mathrm{n}=0)\end{array}$ & $\begin{array}{c}0 \% \\
(\mathrm{n}=0)\end{array}$ & $\begin{array}{l}1.52 \% \\
(\mathrm{n}=1)\end{array}$ & $\begin{array}{c}0 \% \\
(\mathrm{n}=0)\end{array}$ & $\begin{array}{l}0.34 \% \\
(\mathrm{n}=1)\end{array}$ \\
\hline Pegamento, jabón y pasta & $\begin{array}{c}0 \% \\
(\mathrm{n}=0)\end{array}$ & $\begin{array}{c}0 \% \\
(\mathrm{n}=0)\end{array}$ & $\begin{array}{l}1.52 \% \\
(\mathrm{n}=1)\end{array}$ & $\begin{array}{c}0 \% \\
(\mathrm{n}=0)\end{array}$ & $\begin{array}{l}0.34 \% \\
(\mathrm{n}=1)\end{array}$ \\
\hline Papel, jabón y pasta & $\begin{array}{c}0 \% \\
(\mathrm{n}=0)\end{array}$ & $\begin{array}{c}0 \% \\
(\mathrm{n}=0)\end{array}$ & $\begin{array}{c}0 \% \\
(\mathrm{n}=0)\end{array}$ & $\begin{array}{l}1.45 \% \\
(\mathrm{n}=1)\end{array}$ & $\begin{array}{l}0.34 \% \\
(\mathrm{n}=1)\end{array}$ \\
\hline Tierra, gis y pasta & $\begin{array}{c}0 \% \\
(\mathrm{n}=0)\end{array}$ & $\begin{array}{c}0 \% \\
(\mathrm{n}=0)\end{array}$ & $\begin{array}{l}1.52 \% \\
(\mathrm{n}=1)\end{array}$ & $\begin{array}{c}0 \% \\
(\mathrm{n}=0)\end{array}$ & $\begin{array}{l}0.34 \% \\
(\mathrm{n}=1)\end{array}$ \\
\hline
\end{tabular}

Por otra parte, $24.31 \%$ de los participantes afirmó consumir alimentos crudos o no procesados; $16.78 \%$ dijo consumir una sustancia, $5.13 \%$ dos sustancias y $2.40 \%$ tres sustancias. Cabe resaltar que tanto las mujeres en la adolescencia temprana, como los hombres en la adolescencia tardía, fueron los que más casos de consumo reportaron $(\mathrm{n}=20)($ Tabla 4$]$. 
Tabla 4. Porcentaje de consumo de alimentos crudos o no procesados en adolescentes mexicanos.

\begin{tabular}{|c|c|c|c|c|c|}
\hline \multirow{4}{*}{$\begin{array}{c}\text { Consumo de alimentos crudos } \\
\text { o no procesados }\end{array}$} & \multicolumn{2}{|c|}{$\begin{array}{l}\text { Mujeres } \\
(\mathrm{n}=157)\end{array}$} & \multirow{2}{*}{\multicolumn{2}{|c|}{$\begin{array}{l}\text { Hombres } \\
(\mathrm{n}=135)\end{array}$}} & \multirow{4}{*}{$\begin{array}{l}\text { Muestra total } \\
\quad(\mathbf{N}=\mathbf{2 9 2})\end{array}$} \\
\hline & \multicolumn{2}{|c|}{ Adolescencia } & & & \\
\hline & Temprana & Tardía & Temprana & Tardía & \\
\hline & $(\mathrm{n}=85)$ & $(n=72)$ & $(n=66)$ & $(\mathrm{n}=69)$ & \\
\hline Verduras congeladas & $\begin{array}{l}5.88 \% \\
(\mathrm{n}=5)\end{array}$ & $\begin{array}{l}1.39 \% \\
(\mathrm{n}=1)\end{array}$ & $\begin{array}{l}6.06 \% \\
(\mathrm{n}=4)\end{array}$ & $\begin{array}{l}7.25 \% \\
(\mathrm{n}=5)\end{array}$ & $\begin{array}{l}5.14 \% \\
(\mathrm{n}=15)\end{array}$ \\
\hline Arroz o pasta cruda & $\begin{array}{l}4.70 \% \\
(\mathrm{n}=4)\end{array}$ & $\begin{array}{l}4.17 \% \\
(\mathrm{n}=3)\end{array}$ & $\begin{array}{l}9.09 \% \\
(\mathrm{n}=6)\end{array}$ & $\begin{array}{l}2.90 \% \\
(\mathrm{n}=2)\end{array}$ & $\begin{array}{c}5.14 \% \\
(\mathrm{n}=15)\end{array}$ \\
\hline Granos de café & $\begin{array}{l}2.35 \% \\
(\mathrm{n}=2)\end{array}$ & $\begin{array}{l}6.94 \% \\
(\mathrm{n}=5)\end{array}$ & $\begin{array}{l}1.51 \% \\
(\mathrm{n}=1)\end{array}$ & $\begin{array}{l}7.25 \% \\
(\mathrm{n}=5)\end{array}$ & $\begin{array}{c}4.45 \% \\
(n=13)\end{array}$ \\
\hline Bicarbonato & $\begin{array}{l}1.18 \% \\
(\mathrm{n}=1)\end{array}$ & $\begin{array}{l}5.56 \% \\
(\mathrm{n}=4)\end{array}$ & $\begin{array}{l}1.51 \% \\
(\mathrm{n}=1)\end{array}$ & $\begin{array}{c}0 \% \\
(\mathrm{n}=0)\end{array}$ & $\begin{array}{l}2.05 \% \\
(\mathrm{n}=6)\end{array}$ \\
\hline $\begin{array}{l}\text { Verduras congeladas y arroz } \\
\text { o pasta cruda }\end{array}$ & $\begin{array}{l}2.35 \% \\
(\mathrm{n}=2)\end{array}$ & $\begin{array}{l}4.17 \% \\
(\mathrm{n}=3)\end{array}$ & $\begin{array}{c}0 \% \\
(\mathrm{n}=0)\end{array}$ & $\begin{array}{l}5.80 \% \\
(\mathrm{n}=4)\end{array}$ & $\begin{array}{l}3.08 \% \\
(\mathrm{n}=9)\end{array}$ \\
\hline Granos de café y arroz & $\begin{array}{l}4.70 \% \\
(\mathrm{n}=4)\end{array}$ & $\begin{array}{c}0 \% \\
(\mathrm{n}=0)\end{array}$ & $\begin{array}{c}0 \% \\
(\mathrm{n}=0)\end{array}$ & $\begin{array}{c}0 \% \\
(\mathrm{n}=0)\end{array}$ & $\begin{array}{l}1.38 \% \\
(\mathrm{n}=4)\end{array}$ \\
\hline Granos de café y bicarbonato & $\begin{array}{l}1.18 \% \\
(\mathrm{n}=1)\end{array}$ & $\begin{array}{c}0 \% \\
(\mathrm{n}=0)\end{array}$ & $\begin{array}{l}1.51 \% \\
(\mathrm{n}=1)\end{array}$ & $\begin{array}{c}0 \% \\
(\mathrm{n}=0)\end{array}$ & $\begin{array}{l}0.68 \% \\
(\mathrm{n}=2)\end{array}$ \\
\hline $\begin{array}{l}\text { Verduras congeladas, arroz } \\
\text { o pasta cruda y granos de café }\end{array}$ & $\begin{array}{l}1.18 \% \\
(\mathrm{n}=1)\end{array}$ & $\begin{array}{l}1.39 \% \\
(\mathrm{n}=1)\end{array}$ & $\begin{array}{c}0 \% \\
(\mathrm{n}=0)\end{array}$ & $\begin{array}{l}4.35 \% \\
(\mathrm{n}=3)\end{array}$ & $\begin{array}{l}1.71 \% \\
(\mathrm{n}=5)\end{array}$ \\
\hline $\begin{array}{l}\text { Verduras congeladas, arroz } \\
\text { o pasta cruda y bicarbonato }\end{array}$ & $\begin{array}{c}0 \% \\
(\mathrm{n}=0)\end{array}$ & $\begin{array}{c}0 \% \\
(\mathrm{n}=0)\end{array}$ & $\begin{array}{l}1.51 \% \\
(\mathrm{n}=1)\end{array}$ & $\begin{array}{l}1.45 \% \\
(\mathrm{n}=1)\end{array}$ & $\begin{array}{l}0.68 \% \\
(\mathrm{n}=2)\end{array}$ \\
\hline
\end{tabular}

\section{Rumiación}

La conducta de regurgitar fue reportada por $1.71 \%$ de la muestra, del cual 1.38\% indicó realizarla dos o tres veces a la semana. Entre sus causas apun- taron padecer ansiedad $(0.34 \%)$ o enfermedad gastrointestinal $(1.03 \%)$, o bien no poder vomitar $(0.34 \%)$. Cabe señalar que esta conducta es más usual en las mujeres que atraviesan la adolescencia tardía (Tabla 5).

Tabla 5. Porcentaje de presencia y frecuencia de regurgitación en adolescentes mexicanos.

\begin{tabular}{|c|c|c|c|c|c|}
\hline & \multicolumn{2}{|c|}{$\begin{array}{l}\text { Mujeres } \\
(\mathrm{n}=157)\end{array}$} & \multicolumn{2}{|c|}{$\begin{array}{c}\text { Hombres } \\
(n=135)\end{array}$} & \multirow{4}{*}{$\begin{array}{c}\begin{array}{c}\text { Muestra } \\
\text { total }\end{array} \\
(\mathrm{N}=292)\end{array}$} \\
\hline & \multicolumn{4}{|c|}{ Adolescencia } & \\
\hline & Temprana & Tardía & Temprana & Tardía & \\
\hline Regurgitación & $(\mathrm{n}=85)$ & $(\mathrm{n}=72)$ & $(n=66)$ & $(n=69)$ & \\
\hline Si & $\begin{array}{l}1.18 \% \\
(\mathrm{n}=1)\end{array}$ & $\begin{array}{l}4.17 \% \\
(n=3)\end{array}$ & $\begin{array}{l}1.52 \% \\
(\mathrm{n}=1)\end{array}$ & $\begin{array}{c}0 \% \\
(\mathrm{n}=0)\end{array}$ & $\begin{array}{l}1.71 \% \\
(\mathrm{n}=5)\end{array}$ \\
\hline \multicolumn{6}{|l|}{ Frecuencia } \\
\hline Un día a la semana & $\begin{array}{c}0 \% \\
(\mathrm{n}=0)\end{array}$ & $\begin{array}{c}0 \% \\
(\mathrm{n}=0)\end{array}$ & $\begin{array}{l}1.52 \% \\
(\mathrm{n}=1)\end{array}$ & $\begin{array}{c}0 \% \\
(\mathrm{n}=0)\end{array}$ & $\begin{array}{l}0.34 \% \\
(\mathrm{n}=1)\end{array}$ \\
\hline Dos o tres veces por semana & $\begin{array}{l}1.18 \% \\
(\mathrm{n}=1)\end{array}$ & $\begin{array}{l}4.17 \% \\
(n=3)\end{array}$ & $\begin{array}{c}0 \% \\
(\mathrm{n}=0)\end{array}$ & $\begin{array}{c}0 \% \\
(\mathrm{n}=0)\end{array}$ & $\begin{array}{l}1.38 \% \\
(\mathrm{n}=4)\end{array}$ \\
\hline \multicolumn{6}{|l|}{ Causas } \\
\hline Me da ansiedad & $\begin{array}{l}1.18 \% \\
(\mathrm{n}=1)\end{array}$ & $\begin{array}{c}0 \% \\
(\mathrm{n}=0)\end{array}$ & $\begin{array}{c}0 \% \\
(\mathrm{n}=0)\end{array}$ & $\begin{array}{c}0 \% \\
(\mathrm{n}=0)\end{array}$ & $\begin{array}{l}0.34 \% \\
(\mathrm{n}=1)\end{array}$ \\
\hline Sufro una enfermedad gastrointestinal & $\begin{array}{c}0 \% \\
(\mathrm{n}=0)\end{array}$ & $\begin{array}{l}4.16 \% \\
(\mathrm{n}=3)\end{array}$ & $\begin{array}{c}0 \% \\
(\mathrm{n}=0)\end{array}$ & $\begin{array}{c}0 \% \\
(\mathrm{n}=0)\end{array}$ & $\begin{array}{l}1.03 \% \\
(\mathrm{n}=3)\end{array}$ \\
\hline No puedo vomitar & $\begin{array}{c}0 \% \\
(\mathrm{n}=0)\end{array}$ & $\begin{array}{c}0 \% \\
(\mathrm{n}=0)\end{array}$ & $\begin{array}{l}1.52 \% \\
(\mathrm{n}=1)\end{array}$ & $\begin{array}{c}0 \% \\
(\mathrm{n}=0)\end{array}$ & $\begin{array}{l}0.34 \% \\
(\mathrm{n}=1)\end{array}$ \\
\hline
\end{tabular}

Instituto de Investigaciones Psicológicas - Universidad Veracruzana ISSN impreso: 1405-1109
Psicología y Salud, Vol. 31, Núm. 2: 203-214, julio-diciembre de 2021 https://doi.org/10.25009/pys.v31i2.2689 


\section{Evitación/restricción de la ingesta de alimentos (TERIA)}

Respecto a la evitación de alimentos por sus características organolépticas, $24.31 \%$ afirmó no consumir alimentos por el desagrado del olor y el sabor de la comida. En cuanto a la falta de interés por alimentarse, $2.05 \%$ dijo no encontrar disfrute en la comida, y $4.10 \%$ tener poco apetito. Por último, se reportó la evitación por las consecuencias aversivas de los alimentos, donde $0.68 \%$ señaló restringir su consumo por ser dañinos a la salud y $5.82 \%$ para no enfermar.

Tabla 6. Porcentaje de ocurrencia y causas de la reducción de alimentos en adolescentes mexicanos.

\begin{tabular}{|c|c|c|c|c|c|}
\hline \multirow{4}{*}{$\begin{array}{l}\text { Causas de la reducción } \\
\text { en la variedad de alimentos }\end{array}$} & \multicolumn{2}{|c|}{$\begin{array}{l}\text { Mujeres } \\
(\mathrm{n}=157)\end{array}$} & \multicolumn{2}{|c|}{$\begin{array}{l}\text { Hombres } \\
(\mathrm{n}=135)\end{array}$} & \multirow{4}{*}{$\begin{array}{c}\text { Muestra } \\
\text { total } \\
(\mathrm{N}=\mathbf{2 9 2})\end{array}$} \\
\hline & \multicolumn{4}{|c|}{ Adolescencia } & \\
\hline & Temprana & Tardía & Temprana & Tardía & \\
\hline & $(n=85)$ & $(n=72)$ & $(n=66)$ & $(n=69)$ & \\
\hline Me desagrada el olor y sabor & $\begin{array}{l}29.41 \% \\
(\mathrm{n}=25)\end{array}$ & $\begin{array}{l}23.61 \% \\
(\mathrm{n}=17)\end{array}$ & $\begin{array}{l}15.15 \% \\
(\mathrm{n}=10)\end{array}$ & $\begin{array}{l}27.54 \% \\
(\mathrm{n}=19)\end{array}$ & $\begin{array}{l}24.31 \% \\
(\mathrm{n}=71)\end{array}$ \\
\hline Temo subir de peso & $\begin{array}{l}8.24 \% \\
(\mathrm{n}=7)\end{array}$ & $\begin{array}{l}18.06 \% \\
(\mathrm{n}=13)\end{array}$ & $\begin{array}{l}4.55 \% \\
(\mathrm{n}=3)\end{array}$ & $\begin{array}{l}10.14 \% \\
(\mathrm{n}=7)\end{array}$ & $\begin{array}{l}10.27 \% \\
(\mathrm{n}=30)\end{array}$ \\
\hline No me gusta comer & $\begin{array}{l}4.71 \% \\
(\mathrm{n}=4)\end{array}$ & $\begin{array}{l}1.39 \% \\
(\mathrm{n}=1)\end{array}$ & $\begin{array}{c}0 \% \\
(\mathrm{n}=0)\end{array}$ & $\begin{array}{l}1.45 \% \\
(\mathrm{n}=1)\end{array}$ & $\begin{array}{l}2.05 \% \\
(\mathrm{n}=6)\end{array}$ \\
\hline No me da hambre & $\begin{array}{l}7.06 \% \\
(\mathrm{n}=6)\end{array}$ & $\begin{array}{l}1.39 \% \\
(\mathrm{n}=1)\end{array}$ & $\begin{array}{l}4.55 \% \\
(\mathrm{n}=3)\end{array}$ & $\begin{array}{l}2.90 \% \\
(\mathrm{n}=2)\end{array}$ & $\begin{array}{c}4.10 \% \\
(n=12)\end{array}$ \\
\hline No quiero enfermarme & $\begin{array}{l}8.24 \% \\
(\mathrm{n}=7)\end{array}$ & $\begin{array}{l}4.17 \% \\
(\mathrm{n}=3)\end{array}$ & $\begin{array}{l}4.55 \% \\
(\mathrm{n}=3)\end{array}$ & $\begin{array}{l}5.80 \% \\
(\mathrm{n}=4)\end{array}$ & $\begin{array}{l}5.82 \% \\
(\mathrm{n}=17)\end{array}$ \\
\hline Algunos alimentos no son buenos para la salud & $\begin{array}{l}1.18 \% \\
(\mathrm{n}=1)\end{array}$ & $\begin{array}{l}1.39 \% \\
(\mathrm{n}=1)\end{array}$ & $\begin{array}{c}0 \% \\
(\mathrm{n}=0)\end{array}$ & $\begin{array}{c}0 \% \\
(\mathrm{n}=0)\end{array}$ & $\begin{array}{l}0.68 \% \\
(\mathrm{n}=2)\end{array}$ \\
\hline Estoy en tratamiento nutricional & $\begin{array}{l}1.18 \% \\
(\mathrm{n}=1)\end{array}$ & $\begin{array}{l}1.39 \% \\
(\mathrm{n}=1)\end{array}$ & $\begin{array}{c}0 \% \\
(\mathrm{n}=0)\end{array}$ & $\begin{array}{l}4.35 \% \\
(\mathrm{n}=3)\end{array}$ & $\begin{array}{l}1.71 \% \\
(\mathrm{n}=5)\end{array}$ \\
\hline Comía en exceso & $\begin{array}{l}2.35 \% \\
(\mathrm{n}=2)\end{array}$ & $\begin{array}{c}0 \% \\
(\mathrm{n}=0)\end{array}$ & $\begin{array}{l}1.52 \% \\
(\mathrm{n}=1)\end{array}$ & $\begin{array}{c}0 \% \\
(\mathrm{n}=0)\end{array}$ & $\begin{array}{l}1.03 \% \\
(\mathrm{n}=3)\end{array}$ \\
\hline
\end{tabular}

\section{DISCUSIÓN}

El objetivo de la presente investigación fue identificar la ocurrencia de conductas asociadas a pica, trastorno de rumiación y TERIA en adolescentes mexicanos.

Respecto al pica, se encontró que más de una quinta parte de la muestra ingería tanto sustancias no comestibles, como crudas o no procesadas. Este resultado podría ser ligeramente más elevado que el reportado por Hartman et al. (2018) de $12.31 \%$, y por Murray et al. (2018) de $10 \%$, pero menor al del trabajo de Hartman (2019). Lo anterior implica que el consumo de sustancias no comestibles, tanto crudas como procesadas, es más frecuente de lo que se creía. Vale la pena señalar que este comportamiento no tiene que ver en todos los casos con el trastorno de pica, por lo que parece relevante medir otras conductas que permitan detectar con mayor precisión este trastorno.

Asimismo, una cuarta parte de la muestra total afirmó consumir sustancias no comestibles, y un porcentaje ligeramente menor consumía sustancias crudas o no procesadas. Este resultado coincide con lo encontrado por Delaney et al. (2015) y Hartman (2019), quienes afirman que tanto el consumo de sustancias no comestibles (chicles, plástico, tierra), como crudas o no procesadas (comida congelada, pasta cruda y hielo) es frecuente en la población adolescente, lo que indica que el consumo de sustancias no comestibles no es una conducta que únicamente se realice en la infancia, por lo que es necesario seguir indagando sobre la práctica de estas conductas en las diferentes etapas de desarrollo a fin de conocer su evolución y posibles consecuencias en la salud. 
En cuanto al trastorno de rumiación, se evaluó la conducta de regurgitar, hallando que 1.71\% de la muestra total la practicaba. Tal resultado difiere de los de Hartmann et al. (2018) y Murray et al. (2018), quienes reportan $11.94 \%$ y $9.7 \%$, respectivamente. Cabe señalar que en este trabajo también se indagaron los motivos de la aparición de esta conducta, uno de los cuales se debió al padecimiento de enfermedades gastrointestinales, lo que elimina el diagnóstico de trastorno de rumiación, toda vez que la regurgitación no se produce a causa del trastorno en sí sino por dicha afección (APA 2013); también los participantes señalaron la ansiedad como uno de los motivos, lo que parece relevante debido a que una asociación así pudiera ser un eje de investigación para futuros trabajos ya que, como lo señalan Mousa, Montgomery y Alioto (2014), la regurgitación está asociada a eventos estresantes de la vida. La incapacidad de vomitar, fue otra de las causas alegadas, lo que tal vez pudiera estar asociado con el trastorno de bulimia nerviosa debido a que se ha visto que algunas personas que la padecen emplean la regurgitación como método para bajar de peso (Eckern, Stevens y Mitchell, 1998).

Hay que mencionar que las conductas evaluadas relativas al trastorno de evitación/restricción de alimentos fueron la evitación de alimentos como consecuencia de las características organolépticas de los mismos, donde otra cuarta parte de la muestra total los evitaba por el desagrado provocado por su olor y sabor. De igual manera, se reportó la evitación alimentaria por sus consecuencias aversivas, como no enfermarse o porque consideraban que algunos alimentos no eran bue- nos para la salud. Lo anterior coincide con lo dicho por Nicely et al. (2014) en cuanto a que entre las causas de la evitación de alimentos están el temor a sufrir enfermedades como hipercolesterolemia, obesidad o diabetes.

Otra razón aducida para reducir la variedad de alimentos fue el temor a subir de peso; no obstante, esta no es una característica del TERIA en razón de que la restricción alimentaria en este trastorno no se efectúa por cuestiones que involucren la insatisfacción con el peso o con la imagen corporal, como sucede en la anorexia y la bulimia, lo que confirman Fisher et al. (2014), quienes indican que el TERIA es clínicamente diferente a dichos trastornos, y también que los niños y adolescentes que lo padecen son más susceptibles a desarrollar perturbaciones de la alimentación en el futuro, por lo que el diagnóstico y tratamiento del TERIA en edades tempranas resulta fundamental.

En conclusión, este estudio muestra evidencia que apoya que en la adolescencia aparecen conductas asociadas a los trastornos de pica, rumiación y TERIA, como consumir sustancias no comestibles, crudas o no procesadas; hay también regurgitación, evitación de los alimentos por sus características organolépticas, al igual que la reducción de la variedad de alimentos debido a que pudieran provocar enfermedades o ser dañinos para la salud en otras formas.

Algunas limitaciones de este estudio fueron que no se hicieron entrevistas clínicas para corroborar la presencia del trastorno, y que los resultados no pueden ser generalizados a la población porque se dispuso de una muestra no probabilística.

\section{AGRADECIMIENTOS}

Esta investigación se realizó dentro del proyecto IN306518, subvencionado por UNAM-DGAPA-PAPIIT. El primer autor agradece al Consejo Nacional de Ciencia y Tecnología (CONACyT) el apoyo otorgado (920673) para la realización de estudios de doctorado.

Citación: Medina T., K.A., Vázquez A., R., López A., X. y Mancilla D., J.M. (2021). Conductas asociadas a los trastornos de pica, rumiación y evitación/restricción de alimentos en adolescentes. Psicología y Salud, 31(2), 203-214. https://doi.org/10.25009/pys.v31i2.2689. 


\section{REFERENCIAS}

American Psychiatric Association (APA) (1980). Diagnostic and statistical manual of mental disorders (DSM-III). Washington D.C.: American Psychiatric Association.

American Psychiatric Association (APA) (1987). Diagnostic and statistical manual of mental disorders (DSM-III-R). Washington, D.C.: American Psychiatric Association.

American Psychiatric Association (APA) (1994). Diagnostic and statistical manual of mental disorders (DSM-IV). Washington, D.C.: American Psychiatric Association.

American Psychiatric Association (APA) (2000). Diagnostic and statistical manual of mental disorders (DSM-IV-TR). Washington, D.C.: American Psychiatric Association.

American Psychiatric Association (APA) (2013). Diagnostic and statistical manual of mental disorders (DSM-V). Washington: American Psychiatric Association.

Birmingham, C.L. y Firoz, T. (2006). Rumination in eating disorders: Literature review. Eating Weight Disorders, 11, e85-e89.

Bogdanovic, M., Alempijevic, D., Curcic, D. y Durmic, T. (2018). A fatal outcome of pica syndrome: an unusual case of delayed mortality. The American Journal of Forensic Medicine and Pathology, 39(3), 261-263. Doi: 10.1097/ PAF.0000000000000409.

Call, C., Walsh, B.T. y Attia, E. (2013). From DSM-IV to DSM-V: changes to eating disorder diagnoses. Current Opinion in Psychiatry, 26(6), 532-536. Doi: 10.1097/YCO.0b013e328365a321.

Chandran, J.J., Anderson, G., Kennedy, A., Kohn, M. y Clarke, S. (2015). Subacute combined degeneration of the spinal cord in an adolescent male with avoidant/restrictive food intake disorder: A clinical case report. International Journal of Eating Disorders, 48(8), 1176-1179.

Chiarello, F., Marini, E., Ballerini, A. y Ricca, V. (2018). Optic neuropathy due to nutritional deficiency in a male adolescent with avoidant/restrictive food intake disorder: a case report. Eating and Weight Disorders, 23(4), 533-535. Doi: 10.1007/ s40519-017-0409-6.

Danford, D.E. (1982) Pica and nutrition. Annual Review of Nutrition, 2, 303-322.

Delaney, C.B., Eddy, K.T., Hartmann, A.S., Becker, A.E., Murray, H.B. y Thommas, J.J. (2015). Pica and rumination among individuals seeking treatment for eating disorders or obesity. International Journal of Eating Disorders, 48(2), 238-248.

Eckern, M., Stevens, W. y Mitchell, J. (1998). The relationship between rumination and eating disorders. International Journal of Eating Disorders, 26(4), 414-419.

Fisher, M.M., Rosen, D.S., Ornstein, R.M., Mammel, K.A., Katzman, D.K., Rome, E.S., Callahan, S.T., Malizio, J., Kearney, S. y Walsh, B.T. (2014). Characteristics of avoidance restrictive food intake disorder in children and adolescents: A "New Disorder" in DSM-V. Journal of Adolescent Health, 55, 49-52.

Grulee, C.G. (1917). Rumination in the first year of life. American Journal of Diseases of Children, 14(3), 210-218. Doi: 10.1001/ archpedi.1917.01910090055005.

Hartmann, A.S., Poulain, T., Vogel, M., Hiemisch, A., Kiess, W., y Hilbert, A. (2018). Prevalence of pica and rumination behaviors in German children aged 7-14 and their associations with feeding, eating, and general psychopathology: a population-based study. European Child \& Adolescent Psychiatry, 27, 1499-1508.

Imray, J. (1843). Observations on the Mal d'Estomac, or Cachexia Africana, amongst the negroes of Dominica. Edinburgh Medical and Surgical Journal, 6(150), 409.

Malcolm, A., Thumshirn, M.B., Camilleri, M., y Williams, D.E. (1997). Rumination Syndrome. Mayo Clinic Proceedings, 72, 646-652.

Marcus, M.D. y Wildes, J.E. (2016). Eating problems in indviduals with overweight and obesity. En B. T., Walsh, E. Attita, D. R. Glasofer y R. Sysko (Eds.): Handbook of Assessment and Treatment of Eating Disorders (pp. 65-82). Washington, D.C.: American Psychiatric Association.

Medina T., K.A., Vázquez A., R., López A., X. y Mancilla D., J.M. (2017). Conductas de riesgo asociadas a trastornos alimentarios y de la ingestión de alimentos e imagen corporal en adolescentes. Tesis inédita de licenciatura. Ciudad de México: UNAM.

Mousa, H. M., Montgomery, M. y Alioto, A. (2014). Adolescent rumination syndrome. Current Gastroenterology Reports, 16(8), 1-6. Doi: 10.1007/s11894-014-0398-9.

Murray, H.B., Thomas, J.J., Hinz, A., Munsch, S. y Hilbert, A. (2018). Prevalence in primary school youth of pica and rumination behavior: The understudied feeding disorders. International Journal of Eating Disorders, 51(8), 994-998.

Nakai, Y., Nin, K., Noma, S., Teramukai, S. y Wonderlich, S. (2016). Characteristics of avoidance restrictive food intake disorder in a cohort of adult patients. European Eating Disorders Review, 24(6), 528-530. Doi: 10.1002/erv.2476. 
Nakai, Y., Noma, S., Nin, K., Teramukai, S. y Wonderlinch, S.A. (2015). Eating disorder behaviors and attitudes in Japanese adolescents girls and boys in high schools. Psychiatry Research, 230, 722-724.

Nicely, T.A., Lane-Loney, S., Masciulli, E., Hollenbeak, C.S. y Ornstein, R.M. (2014). Prevalence and characteristics of avoidant/ restrictive food intake disorder in a cohort of young patients in day treatment for eating disorders. International Journal of Eating Disorders, 2(21), 2-8.

Norris, M.L. y Katzman, D.K. (2015) Change is never easy, but is posible: Reflections on avoidant/restrictive food intake disorder two year after its introduction in the DSM-5. Journal of Adolescent Health, 57, 8-9.

Norris, M.L., Robinson, A., Obeid, N., Harrison, M., Espettigue, W. y Henderson, K. (2014). Exploring avoidant-restrictive food intake disorder in eating disordered patients: a descriptive study. International Journal of Eating Disorder, 47(5), 495-499.

Parry-Jones, B. (1994). Merycism or rumination disorder. A historical investigation and current assessment. The British Journal of Psychiatry, 165, 303-314.

Parry-Jones, B. y Parry Jones, W. (1992). Pica: symptom or eating disorder? A historical assessment. The British Journal of Psychiatry, 160, 341-354.

Sturmey, P. y Williams, D.E. (2016) Epidemiology. En J. Matson (Ed.): Pica in individuals with developmental disabilities (pp.19-28). New York: Springer. Doi: 10.1007/978-3-319-30798-5.

Uher, R. y Rutter, M. (2012). Classification of feeding and eating disorders: review of evidence and proposals for ICD-11. International Review of Psychiatry, 24(6), 514-529.

Vázquez, R., López, X., Tellez-Girón, M.T. y Mancilla D., J.M. (2015). El diagnóstico de los trastornos alimentarios del DSM-IVTR al DSM-5. Revista Mexicana de Trastornos Alimentarios, 6(2), 108-120. 